Kragujevac Journal of Mathematics

Volume 39(2) (2015), Pages 193-205.

\title{
LAPLACIAN ENERGY OF UNION AND CARTESIAN PRODUCT AND LAPLACIAN EQUIENERGETIC GRAPHS
}

\author{
HARISHCHANDRA S. RAMANE ${ }^{1}$, GOURAMMA A. GUDODAGI ${ }^{1}$, AND IVAN GUTMAN ${ }^{2}$
}

\begin{abstract}
The Laplacian energy of a graph $G$ with $n$ vertices and $m$ edges is defined as $L E(G)=\sum_{i=1}^{n}\left|\mu_{i}-2 m / n\right|$, where $\mu_{1}, \mu_{2}, \ldots, \mu_{n}$ are the Laplacian eigenvalues of $G$. If two graphs $G_{1}$ and $G_{2}$ have equal average vertex degrees, then $L E\left(G_{1} \cup G_{2}\right)=L E\left(G_{1}\right)+L E\left(G_{2}\right)$. Otherwise, this identity is violated. We determine a term $\Xi$, such that $L E\left(G_{1}\right)+L E\left(G_{2}\right)-\Xi \leq L E\left(G_{1} \cup G_{2}\right) \leq$ $L E\left(G_{1}\right)+L E\left(G_{2}\right)+\Xi$ holds for all graphs. Further, by calculating $L E$ of the Cartesian product of some graphs, we construct new classes of Laplacian non-cospectral, Laplacian equienergetic graphs.
\end{abstract}

\section{INTRODUCTION}

Let $G$ be a finite, simple, undirected graph with $n$ vertices $v_{1}, v_{2}, \ldots, v_{n}$ and $m$ edges. In what follows, we say that $G$ is an $(n, m)$-graph. Let $A(G)$ be the adjacency matrix of $G$ and let $\lambda_{1}, \lambda_{2}, \ldots, \lambda_{n}$ be its eigenvalues.

Let $D(G)$ be the diagonal matrix whose $(i, i)$-th entry is the degree of a vertex $v_{i}$. The matrix $C(G)=D(G)-A(G)$ is called the Laplacian matrix of $G$. The Laplacian polynomial of $G$ is defined as $\psi(G, \mu)=\operatorname{det}[\mu I-C(G)]$, where $I$ is an identity matrix. The eigenvalues of $C(G)$, denoted by $\mu_{i}=\mu_{i}(G), i=1,2, \ldots, n$, are called the Laplacian eigenvalues of $G$ [16]. Two graphs are said to be Laplacian cospectral if they have same Laplacian eignevalues. The adjacency eigenvalues and Laplacian eigenvalues satisfies the following conditions:

$$
\sum_{i=1}^{n} \lambda_{i}=0 \quad \text { and } \quad \sum_{i=1}^{n} \mu_{i}=2 m .
$$

Key words and phrases. Laplacian spectrum, Laplacian energy, Cartesian product, Laplacian equienergetic graphs.

2010 Mathematics Subject Classification. Primary: 05C50, 05C75

Received: September 22, 2015.

Accepted: November 11, 2015. 
The energy of a graph $G$ is defined as

$$
\mathcal{E}=\mathcal{E}(G)=\sum_{i=1}^{n}\left|\lambda_{i}\right| .
$$

It was introduced by one of the present authors in the 1970s, and since then has been much studied in both chemical and mathematical literature. For details see the book [15] and the references cited therein.

The Laplacian energy of a graph was introduced a few years ago [13] and is defined as

$$
L E(G)=\sum_{i=1}^{n}\left|\mu_{i}(G)-\frac{2 m}{n}\right| .
$$

This definition is chosen so as to preserve the main features of the ordinary graph energy $\mathcal{E}$, see [18]. Basic properties and other results on Laplacian energy can be found in the survey [1], the recent papers $[6-8,11,12,17,19]$, and the references cited therein.

\section{LAPLACiAn Energy of Union of GraphS}

Let $G_{1}$ and $G_{2}$ be two graphs with disjoint vertex sets. Let for $i=1,2$, the vertex and edges sets of $G_{i}$ be, respectively, $V_{i}$ and $E_{i}$. The union of $G_{1}$ and $G_{2}$ is a graph $G_{1} \cup G_{2}$ with vertex set $V_{1} \cup V_{2}$ and the edge set $E_{1} \cup E_{2}$. If $G_{1}$ is an $\left(n_{1}, m_{1}\right)$-graph and $G_{2}$ is an $\left(n_{2}, m_{2}\right)$-graph then $G_{1} \cup G_{2}$ has $n_{1}+n_{2}$ vertices and $m_{1}+m_{2}$ edges. It is easy to see that the Laplacian spectrum of $G_{1} \cup G_{2}$ is the union of the Laplacian spectra of $G_{1}$ and $G_{2}$.

In [13] it was proven that if $G_{1}$ and $G_{2}$ have equal average vertex degrees, then $L E\left(G_{1} \cup G_{2}\right)=L E\left(G_{1}\right)+L E\left(G_{2}\right)$. If the average vertex degrees are not equal, that is $\frac{2 m_{1}}{n_{1}} \neq \frac{2 m_{2}}{n_{2}}$, then it may be either $L E\left(G_{1} \cup G_{2}\right)>L E\left(G_{1}\right)+L E\left(G_{2}\right)$ or $L E\left(G_{1} \cup G_{2}\right)<L E\left(G_{1}\right)+L E\left(G_{2}\right)$ or, exceptionally, $L E\left(G_{1} \cup G_{2}\right)=L E\left(G_{1}\right)+L E\left(G_{2}\right)$ [13].

In this section we study the Laplacian establish some additional relations between $L E\left(G_{1} \cup G_{2}\right)$ and $L E\left(G_{1}\right)+L E\left(G_{2}\right)$.

Theorem 2.1. Let $G_{1}$ be an $\left(n_{1}, m_{1}\right)$-graph and $G_{2}$ be an $\left(n_{2}, m_{2}\right)$-graph, such that $\frac{2 m_{1}}{n_{1}}>\frac{2 m_{2}}{n_{2}}$. Then

$$
L E\left(G_{1}\right)+L E\left(G_{2}\right)-\frac{4\left(n_{2} m_{1}-n_{1} m_{2}\right)}{n_{1}+n_{2}} \leq L E\left(G_{1} \cup G_{2}\right)
$$

$$
\leq L E\left(G_{1}\right)+L E\left(G_{2}\right)+\frac{4\left(n_{2} m_{1}-n_{1} m_{2}\right)}{n_{1}+n_{2}} .
$$


Proof. For the sake of simplicity, denote $G_{1} \cup G_{2}$ by $G$. Then $G$ is an $\left(n_{1}+n_{2}, m_{1}+m_{2}\right)$ graphs. By the definition of Laplacian energy,

$$
\begin{aligned}
L E\left(G_{1} \cup G_{2}\right)= & \sum_{i=1}^{n_{1}+n_{2}}\left|\mu_{i}(G)-\frac{2\left(m_{1}+m_{2}\right)}{n_{1}+n_{2}}\right| \\
= & \sum_{i=1}^{n_{1}}\left|\mu_{i}(G)-\frac{2\left(m_{1}+m_{2}\right)}{n_{1}+n_{2}}\right|+\sum_{i=n_{1}+1}^{n_{1}+n_{2}}\left|\mu_{i}(G)-\frac{2\left(m_{1}+m_{2}\right)}{n_{1}+n_{2}}\right| \\
= & \sum_{i=1}^{n_{1}}\left|\mu_{i}\left(G_{1}\right)-\frac{2\left(m_{1}+m_{2}\right)}{n_{1}+n_{2}}\right|+\sum_{i=1}^{n_{2}}\left|\mu_{i}\left(G_{2}\right)-\frac{2\left(m_{1}+m_{2}\right)}{n_{1}+n_{2}}\right| \\
= & \sum_{i=1}^{n_{1}}\left|\mu_{i}\left(G_{1}\right)-\frac{2 m_{1}}{n_{1}}+\frac{2 m_{1}}{n_{1}}-\frac{2\left(m_{1}+m_{2}\right)}{n_{1}+n_{2}}\right| \\
& +\sum_{i=1}^{n_{2}}\left|\mu_{i}\left(G_{2}\right)-\frac{2 m_{2}}{n_{2}}+\frac{2 m_{2}}{n_{2}}-\frac{2\left(m_{1}+m_{2}\right)}{n_{1}+n_{2}}\right| \\
\leq & \sum_{i=1}^{n_{1}}\left|\mu_{i}\left(G_{1}\right)-\frac{2 m_{1}}{n_{1}}\right|+n_{1}\left|\frac{2 m_{1}}{n_{1}}-\frac{2\left(m_{1}+m_{2}\right)}{n_{1}+n_{2}}\right| \\
& +\sum_{i=1}^{n_{2}}\left|\mu_{i}\left(G_{2}\right)-\frac{2 m_{2}}{n_{2}}\right|+n_{2}\left|\frac{2 m_{2}}{n_{2}}-\frac{2\left(m_{1}+m_{2}\right)}{n_{1}+n_{2}}\right| .
\end{aligned}
$$

Since $n_{2} m_{1}>n_{1} m_{2}$, Eq. (2.2) becomes

$$
\begin{aligned}
L E\left(G_{1} \cup G_{2}\right) \leq & L E\left(G_{1}\right)+n_{1}\left(\frac{2 m_{1}}{n_{1}}-\frac{2\left(m_{1}+m_{2}\right)}{n_{1}+n_{2}}\right) \\
& +\operatorname{LE}\left(G_{2}\right)+n_{2}\left(-\frac{2 m_{2}}{n_{2}}+\frac{2\left(m_{1}+m_{2}\right)}{n_{1}+n_{2}}\right) \\
= & L E\left(G_{1}\right)+\operatorname{LE}\left(G_{2}\right)+\frac{4\left(n_{2} m_{1}-n_{1} m_{2}\right)}{n_{1}+n_{2}}
\end{aligned}
$$

which is an upper bound.

To obtain the lower bound, we just have to note that in full analogy to the above arguments,

$$
L E\left(G_{1} \cup G_{2}\right) \geq \sum_{i=1}^{n_{1}}\left|\mu_{i}\left(G_{1}\right)-\frac{2 m_{1}}{n_{1}}\right|-n_{1}\left|\frac{2 m_{1}}{n_{1}}-\frac{2\left(m_{1}+m_{2}\right)}{n_{1}+n_{2}}\right|
$$




$$
+\sum_{i=1}^{n_{2}}\left|\mu_{i}\left(G_{2}\right)-\frac{2 m_{2}}{n_{2}}\right|-n_{2}\left|\frac{2 m_{2}}{n_{2}}-\frac{2\left(m_{1}+m_{2}\right)}{n_{1}+n_{2}}\right| .
$$

Since $n_{2} m_{1}>n_{1} m_{2}$, the Eq. (2.3) becomes

$$
\begin{aligned}
L E\left(G_{1} \cup G_{2}\right) \geq & L E\left(G_{1}\right)-n_{1}\left(\frac{2 m_{1}}{n_{1}}-\frac{2\left(m_{1}+m_{2}\right)}{n_{1}+n_{2}}\right) \\
& +\operatorname{LE}\left(G_{2}\right)-n_{2}\left(-\frac{2 m_{2}}{n_{2}}+\frac{2\left(m_{1}+m_{2}\right)}{n_{1}+n_{2}}\right) \\
= & L E\left(G_{1}\right)+\operatorname{LE}\left(G_{2}\right)-\frac{4\left(n_{2} m_{1}-n_{1} m_{2}\right)}{n_{1}+n_{2}}
\end{aligned}
$$

which is a lower bound.

Corollary 2.1. [13] Let $G_{1}$ be an $\left(n_{1}, m_{1}\right)$-graph and $G_{2}$ be $\left(n_{2}, m_{2}\right)$-graph such that $\frac{2 m_{1}}{n_{1}}=\frac{2 m_{2}}{n_{2}}$. Then

$$
L E\left(G_{1} \cup G_{2}\right)=L E\left(G_{1}\right)+L E\left(G_{2}\right) .
$$

Corollary 2.2. Let $G_{1}$ be an $r_{1}$-regular graph on $n_{1}$ vertices and $G_{2}$ be an $r_{2}$-regular graph on $n_{2}$ vertices, such that $r_{1}>r_{2}$. Then

$$
\begin{aligned}
L E\left(G_{1}\right)+L E\left(G_{2}\right)-\frac{2 n_{1} n_{2}\left(r_{1}-r_{2}\right)}{n_{1}+n_{2}} & \leq L E\left(G_{1} \cup G_{2}\right) \\
& \leq L E\left(G_{1}\right)+L E\left(G_{2}\right)+\frac{2 n_{1} n_{2}\left(r_{1}-r_{2}\right)}{n_{1}+n_{2}} .
\end{aligned}
$$

Proof. Result follows by setting $m_{1}=n_{1} r_{1} / 2$ and $m_{2}=n_{2} r_{2} / 2$ into Theorem 2.1.

Theorem 2.2. Let $G$ be an $(n, m)$-graph and $\bar{G}$ be its complement, and let $m>$ $n(n-1) / 4$. Then

$$
L E(G)+L E(\bar{G})-[4 m-n(n-1)] \leq L E(G \cup \bar{G}) \leq L E(G)+L E(\bar{G})+[4 m-n(n-1)] .
$$

Proof. $\bar{G}$ is a graph with $n$ vertices and $n(n-1) / 2-m$ edges. Substituting this into Eq. (2.1), the result follows.

Theorem 2.3. Let $G$ be an $(n, m)$-graph and $G^{\prime}$ be the graph obtained from $G$ by removing $k$ edges, $0 \leq k \leq m$. Then

$$
L E(G)+L E\left(G^{\prime}\right)-2 k \leq L E\left(G \cup G^{\prime}\right) \leq L E(G)+L E\left(G^{\prime}\right)+2 k .
$$

Proof. The number of vertices of $G^{\prime}$ is $n$ and the number of edges is $m-k$. Substituting this in Eq. (2.1), the result follows. 


\section{Laplacian Energy of Cartesian Product}

Let $G$ be a graph with vertex set $V_{1}$ and $H$ be a graph with vertex set $V_{2}$. The Cartesian product of $G$ and $H$, denoted by $G \times H$ is a graph with vertex set $V_{1} \times V_{2}$, such that two vertices $\left(u_{1}, v_{1}\right)$ and $\left(u_{2}, v_{2}\right)$ are adjacent in $G \times H$ if and only if either $u_{1}=u_{2}$ and $v_{1}$ is adjacent to $v_{2}$ in $H$ or $v_{1}=v_{2}$ and $u_{1}$ is adjacent to $u_{2}$ in $G$ [14].

Lemma 3.1. [9] Let $A=\left[\begin{array}{cc}A_{0} & A_{1} \\ A_{1} & A_{0}\end{array}\right]$ be a symmetric $2 \times 2$ block matrix. Then the spectrum of $A$ is the union of the spectra of $A_{0}+A_{1}$ and $A_{0}-A_{1}$.

Theorem 3.1. If $\mu_{1}, \mu_{2}, \ldots, \mu_{n}$ are the Laplacian eigenvalues of a graph $G$, then the Laplacian eigenvalues of $G \times K_{2}$ are $\mu_{1}, \mu_{2}, \ldots, \mu_{n}$ and $\mu_{1}+2, \mu_{2}+2, \ldots, \mu_{n}+2$.

Proof. The Laplacian matrix of $G \times K_{2}$ is

$$
C\left(G \times K_{2}\right)=\left[\begin{array}{cc}
C(G)+I & -I \\
-I & C(G)+I
\end{array}\right]=\left[\begin{array}{ll}
C_{0} & C_{1} \\
C_{1} & C_{0}
\end{array}\right]
$$

where $C(G)$ is the Laplacian matrix of $G$ and $I$ is an identity matrix of order $n$. By Lemma 3.1, the Laplacian spectrum of $G \times K_{2}$ is the union of the spectra of $C_{0}+C_{1}$ and $C_{0}-C_{1}$.

Here $C_{0}+C_{1}=C(G)$. Therefore, the eigenvalues of $C_{0}+C_{1}$ are the Laplacian eigenvalues of $G$.

Because $C_{0}-C_{1}=C(G)+2 I$, the characteristic polynomial of $C_{0}-C_{1}$ is

$$
\begin{aligned}
\psi\left(C_{0}-C_{1}, \mu\right) & =\operatorname{det}\left[\mu I-\left(C_{0}-C_{1}\right)\right]=\operatorname{det}[\mu I-(C(G)+2 I)] \\
& =\operatorname{det}[(\mu-2) I-C(G))]=\psi(G, \mu-2) .
\end{aligned}
$$

Therefore the eigenvalues of $C_{0}-C_{1}$ are $\mu_{i}+2, i=1,2, \ldots, n$.

The Laplacian eigenvalues of the complete graph $K_{n}$ are $n(n-1$ times) and 0 . The Laplacian eigenvalues of the complete bipartite regular graph $K_{k, k}$ are $2 k, k(2 k-2$ times) and 0 . The Laplacian eigenvalues of the cocktail party graph $C P(k)$ (the regular graph on $n=2 k$ vertices and of degree $2 k-2)$ are $2 k$ ( $k-1$ times), $2 k-2(k$ times) and 0 [16]. Applying Theorem 3.1, we directly arrive at the following example.

Example 3.1.

$$
\begin{aligned}
& L E\left(K_{n} \times K_{2}\right)= \begin{cases}4 n-4, & \text { if } n>2, \\
2 n, & \text { if } n \leq 2,\end{cases} \\
& L E\left(K_{k, k} \times K_{2}\right)= \begin{cases}8 k-4, & \text { if } k>1, \\
6 k-2, & \text { if } k=1,\end{cases} \\
& L E\left(C P(k) \times K_{2}\right)=10 k-8, \quad \text { if } k \geq 2 .
\end{aligned}
$$

Theorem 3.2. Let $G$ be an $(n, m)$-graph. Then

$$
2[L E(G)-n] \leq L E\left(G \times K_{2}\right) \leq 2[L E(G)+n] .
$$


Proof. Let $\mu_{1}, \mu_{2}, \ldots, \mu_{n}$ be the Laplacian eigenvalues of $G$. Then by Theorem 3.1, the Laplacian eigenvalues of $G \times K_{2}$ are $\mu_{i}, i=1,2, \ldots, n$ and $\mu_{i}+2, i=1,2, \ldots, n$. The graph $G \times K_{2}$ has $2 n$ vertices and $2 m+n$ edges. Therefore,

$$
\begin{aligned}
L E\left(G \times K_{2}\right) & =\sum_{i=1}^{n}\left|\mu_{i}-\frac{2(2 m+n)}{2 n}\right|+\sum_{i=1}^{n}\left|\mu_{i}+2-\frac{2(2 m+n)}{2 n}\right| \\
& =\sum_{i=1}^{n}\left|\mu_{i}-\frac{2 m}{n}-1\right|+\sum_{i=1}^{n}\left|\mu_{i}-\frac{2 m}{n}+1\right| .
\end{aligned}
$$

Equation (3.1) can be rewritten as

$$
L E\left(G \times K_{2}\right) \leq \sum_{i=1}^{n}\left|\mu_{i}-\frac{2 m}{n}\right|+n+\sum_{i=1}^{n}\left|\mu_{i}-\frac{2 m}{n}\right|+n=2 L E(G)+2 n
$$

which is an upper bound.

For lower bound, Eq. (3.1) can be rewritten as

$$
L E\left(G \times K_{2}\right) \geq \sum_{i=1}^{n}\left|\mu_{i}-\frac{2 m}{n}\right|-n+\sum_{i=1}^{n}\left|\mu_{i}-\frac{2 m}{n}\right|-n=2 L E(G)-2 n .
$$

Theorem 3.3. For a graph $G$ with $n$ vertices, $L E\left(G \times K_{2}\right) \geq 2 n$.

Proof. From Eq. (3.1)

$$
\begin{aligned}
L E\left(G \times K_{2}\right) & \geq\left|\sum_{i=1}^{n}\left(\mu_{i}-\frac{2 m}{n}-1\right)\right|+\left|\sum_{i=1}^{n}\left(\mu_{i}-\frac{2 m}{n}+1\right)\right| \\
& =|2 m-2 m-n|+|2 m-2 m+n|=2 .
\end{aligned}
$$

\section{LAPLACIAN EQUIENERGETIC GRAPHS}

Two graphs $G_{1}$ and $G_{2}$ are said to be equienergetic if $\mathcal{E}\left(G_{1}\right)=\mathcal{E}\left(G_{2}\right)$ [2]. For details see the book [15]. In analogy to this, two graphs $G_{1}$ and $G_{2}$ are said to be Laplacian equienergetic if $L E\left(G_{1}\right)=L E\left(G_{2}\right)$.

Obviously Laplacian cospectral graphs are Laplacian equienergetic. Therefore we are interested in Laplacian non-cospectral graphs with equal number of vertices, having equal Laplacian energies. Stevanović [24] has constructed Laplacian equienergetic threshold graphs. Fritscher et al. [10] discovered a family of Laplacian equienergetic unicyclic graphs. We now report some additional classes of such graphs.

The line graph of the graph $G$, denoted by $L(G)$, is a graph whose vertices corresponds to the edges of $G$ and two vertices in $L(G)$ are adjacent if and only if the corresponding edges are adjacent in $G$ [14]. The $k$-th iterated line graph of $G$ is defined 
as $L^{k}(G)=L\left(L^{k-1}(G)\right)$ where $L^{0}(G) \equiv G$ and $L^{1}(G) \equiv L(G)$. If $G$ is a regular graph of order $n_{0}$ and of degree $r_{0}$, then $L(G)$ is a regular graph of order $n_{1}=n_{0} r_{0} / 2$ and of degree $r_{1}=2 r_{0}-2$. Consequently, the order and degree of $L^{k}(G)$ are $[3,4]$ :

$$
n_{k}=\frac{1}{2} n_{k-1} r_{k-1} \quad \text { and } \quad r_{k}=2 r_{k-1}-2
$$

where $n_{i}$ and $r_{i}$ stand for the order and degree of $L^{i}(G), i=0,1,2, \ldots$ Therefore $[3,4]$,

$$
n_{k}=\frac{n_{0}}{2^{k}} \prod_{i=0}^{k-1} r_{i}=\frac{n_{0}}{2^{k}} \prod_{i=0}^{k-1}\left(2^{i} r_{0}-2^{i+1}+2\right)
$$

and

$$
r_{k}=2^{k} r_{0}-2^{k+1}+2 \text {. }
$$

Theorem 4.1. [23] If $\lambda_{1}, \lambda_{2}, \ldots, \lambda_{n}$ are the adjacency eigenvalues of a regular graph $G$ of order $n$ and of degree $r$, then the adjacency eigenvalues of $L(G)$ are

$$
\begin{array}{rl}
\lambda_{i}+r-2 & i=1,2, \ldots, n, \quad \text { and } \\
-2 & n(r-2) / 2 \text { times. }
\end{array}
$$

Theorem 4.2. [22] If $\lambda_{1}, \lambda_{2}, \ldots, \lambda_{n}$ are the adjacency eigenvalues of a regular graph $G$ of order $n$ and of degree $r$, then the adjacency eigenvalues of $\bar{G}$, the complement of $G$, are $n-r-1$ and $-\lambda_{i}-1, i=2,3, \ldots, n$.

Theorem 4.3. [16] If $\lambda_{1}, \lambda_{2}, \ldots, \lambda_{n}$ are the adjacency eigenvalues of a regular graph $G$ of order $n$ and of degree $r$, then its Laplacian eigenvalues are $r-\lambda_{i}, i=1,2, \ldots, n$.

For $G$ being a regular graph of degree $r \geq 3$, and for $k \geq 2$, expressions for $\mathcal{E}\left(L^{k}(G)\right)$ and $\mathcal{E}\left(\overline{L^{k}(G)}\right)$ were reported in $[20,21]$.

Theorem 4.4. If $G$ is a regular graph of order $n$ and of degree $r \geq 4$, then

$$
L E\left(L^{2}(G) \times K_{2}\right)=4 n r(r-2) .
$$

Proof. Let $\lambda_{1}, \lambda_{2}, \ldots, \lambda_{n}$ be the adjacency eigenvalues of $G$. Then by Theorem 4.1, the adjacency eigenvalues of $L(G)$ are

$$
\left.\begin{array}{rl}
\lambda_{i}+r-2 & i=1,2, \ldots, n, \quad \text { and } \\
-2 & n(r-2) / 2 \text { times. }
\end{array}\right\}
$$

Since $L(G)$ is a regular graph of order $n r / 2$ and of degree $2 r-2$, by Eq. (4.3) the adjacency eigenvalues of $L^{2}(G)$ are

$$
\left.\begin{array}{rlr}
\lambda_{i}+3 r-6 & i=1,2, \ldots, n, & \text { and } \\
2 r-6 & n(r-2) / 2 \text { times, } & \text { and } \\
-2 & n r(r-2) / 2 \text { times. }
\end{array}\right\}
$$


Since $L^{2}(G)$ is a regular graph of order $n r(r-1) / 2$ and of degree $4 r-2$, by Theorem 4.3 and Eq. (4.4), the Laplacian eigenvalues of $L^{2}(G)$ are

$$
\left.\begin{array}{rll}
r-\lambda_{i} & i=1,2, \ldots, n, & \text { and } \\
2 r & n(r-2) / 2 \text { times, } & \text { and } \\
4 r-4 & n r(r-2) / 2 \text { times. } &
\end{array}\right\}
$$

Using Theorem 3.1 and Eq. (4.5), the Laplacian eigenvalues of $L^{2}(G) \times K_{2}$ are

$$
\begin{array}{rlr}
r-\lambda_{i} & i=1,2, \ldots, n, & \text { and } \\
2 r & n(r-2) / 2 \text { times, } & \text { and } \\
4 r-4 & n r(r-2) / 2 \text { times, } & \text { and } \\
r-\lambda_{i}+2 & i=1,2, \ldots, n, & \text { and } \\
2 r+2 & n(r-2) / 2 \text { times, } & \text { and } \\
4 r-2 & n r(r-2) / 2 \text { times. } &
\end{array}
$$

The graph $L^{2}(G) \times K_{2}$ is a regular graph of order $n r(r-1)$ and of degree $4 r-5$. By Eq. (4.6), the Laplacian energy of $L^{2}(G) \times K_{2}$ is computed as

$$
\begin{aligned}
L E\left(L^{2}(G) \times K_{2}\right)= & \sum_{i=1}^{n}\left|r-\lambda_{i}-(4 r-5)\right|+|2 r-(4 r-5)| \frac{n(r-2)}{2} \\
& +|4 r-4-(4 r-5)| \frac{n r(r-2)}{2}+\sum_{i=1}^{n}\left|r-\lambda_{i}+2-(4 r-5)\right| \\
& +|2 r+2-(4 r-5)| \frac{n(r-2)}{2}+|4 r-2-(4 r-5)| \frac{n r(r-2)}{2} \\
= & \sum_{i=1}^{n}\left|-\lambda_{i}-3 r+5\right|+|-2 r+5| \frac{n(r-2)}{2} \\
& +|1| \frac{n r(r-2)}{2}+\sum_{i=1}^{n}\left|-\lambda_{i}-3 r+7\right| \\
& +|-2 r+7| \frac{n(r-2)}{2}+|3| \frac{n r(r-2)}{2} .
\end{aligned}
$$

If $d_{\text {max }}$ is the greatest vertex degree of a graph, then all its adjacency eigenvalues belongs to the interval $\left[-d_{\max },+d_{\max }\right]$ [5]. In particular, the adjacency eigenvalues of a regular graph of degree $r$ satisfy the condition $-r \leq \lambda_{i} \leq r, i=1,2, \ldots, n$. 
If $r \geq 4$ then $\lambda_{i}+3 r-5>0, \lambda_{i}+3 r-7>0,2 r-5>0$, and $2 r-7>0$. Therefore by Eq. (4.7), and bearing in mind that $\sum_{i=1}^{n} \lambda_{i}=0$,

$$
\begin{aligned}
L E\left(L^{2}(G) \times K_{2}\right)= & \sum_{i=1}^{n} \lambda_{i}+n(3 r-5)+(2 r-5) \frac{n(r-2)}{2}+\frac{n r(r-2)}{2} \\
& +\sum_{i=1}^{n} \lambda_{i}+n(3 r-7)+(2 r-7) \frac{n(r-2)}{2}+\frac{3 n r(r-2)}{2} \\
= & 4 n r(r-2) .
\end{aligned}
$$

Corollary 4.1. Let $G$ be a regular graph of order $n_{0}$ and of degree $r_{0} \geq 4$. Let $n_{k}$ and $r_{k}$ be the order and degree, respectively of the $k$-th iterated line graph $L^{k}(G)$ of $G$, $k \geq 2$. Then

$$
\begin{aligned}
& L E\left(L^{k}(G) \times K_{2}\right)=4 n_{k-2} r_{k-2}\left(r_{k-2}-2\right)=4 n_{k-1}\left(r_{k-1}-2\right), \\
& L E\left(L^{k}(G) \times K_{2}\right)=4 n_{0}\left(r_{0}-2\right) \prod_{i=0}^{k-2}\left(2^{i} r_{0}-2^{i+1}+2\right), \\
& L E\left(L^{k}(G) \times K_{2}\right)=8\left(n_{k}-n_{k-1}\right)=8 n_{k}\left(\frac{r_{k}-2}{r_{k}+2}\right) .
\end{aligned}
$$

From Eq. (4.8) we see that the energy of $L^{k}(G) \times K_{2}, k \geq 2$ is fully determined by the order $n$ and degree $r \geq 4$ of $G$.

Theorem 4.5. If $G$ is a regular graph of order $n$ and of degree $r \geq 3$, then

$$
L E\left(\overline{L^{2}(G)} \times K_{2}\right)=(n r-4)(4 r-6)-4 .
$$

Proof. Let $\lambda_{1}, \lambda_{2}, \ldots, \lambda_{n}$ be the adjacency eigenvalues of a regular graph $G$ of order $n$ and of degree $r \geq 3$. Then the adjacency eigenvalues of $L^{2}(G)$ are as given by Eq. (4.4).

Since $L^{2}(G)$ is a regular graph of order $n r(r-1) / 2$ and of degree $4 r-2$, by Theorem 4.2 and Eq. (4.4), the adjacency eigenvalues of $\overline{L^{2}(G)}$ are

$$
\left.\begin{array}{rlr}
-\lambda_{i}-3 r+5 & i=2,3, \ldots, n, & \text { and } \\
-2 r+5 & n(r-2) / 2 \text { times, } & \text { and } \\
1 & n r(r-2) / 2 \text { times, } & \text { and } \\
(n r(r-1) / 2)-4 r+5 . &
\end{array}\right\}
$$


Since $\overline{L^{2}(G)}$ is a regular graph of order $n r(r-1) / 2$ and of degree $(n r(r-1) / 2)-4 r+5$, by Theorem 4.3 and Eq. (4.9), the Laplacian eigenvalues of $\overline{L^{2}(G)}$ are

$$
\left.\begin{array}{rll}
(n r(r-1) / 2)-r-\lambda_{i} & i=2,3, \ldots, n, & \text { and } \\
(n r(r-1) / 2)-2 r & n(r-2) / 2 \text { times, } & \text { and } \\
(n r(r-1) / 2)-4 r+4 & n r(r-2) / 2 \text { times, } & \text { and } \\
0 . &
\end{array}\right\}
$$

Using Theorem 3.1 and Eq. (4.10), the Laplacian eigenvalues of $\overline{L^{2}(G)} \times K_{2}$ are

$$
\left.\begin{array}{rlr}
(n r(r-1) / 2)-r-\lambda_{i} & i=2,3, \ldots, n, & \text { and } \\
(n r(r-1) / 2)-2 r & n(r-2) / 2 \text { times, } & \text { and } \\
(n r(r-1) / 2)-4 r+4 & n r(r-2) / 2 \text { times, } & \text { and } \\
0 & 1 \text { time, } & \text { and } \\
(n r(r-1) / 2)-r-\lambda_{i}+2 & i=2,3, \ldots, n, & \text { and } \\
(n r(r-1) / 2)-2 r+2 & n(r-2) / 2 \text { times, } & \text { and } \\
(n r(r-1) / 2)-4 r+6 & n r(r-2) / 2 \text { times, } & \text { and } \\
2 . &
\end{array}\right\}
$$

The graph $\overline{L^{2}(G)} \times K_{2}$ is a regular graph of order $n r(r-1)$ and of degree $(n r(r-1) / 2)-4 r+6$. By Eq. (4.11),

$$
\begin{aligned}
L E\left(\overline{L^{2}(G)} \times K_{2}\right)= & \sum_{i=2}^{n}\left|\frac{n r(r-1)}{2}-r+\lambda_{i}-\left(\frac{n r(r-1)}{2}-4 r+6\right)\right| \\
& +\left|\frac{n r(r-1)}{2}-2 r-\left(\frac{n r(r-1)}{2}-4 r+6\right)\right| \frac{n(r-2)}{2} \\
& +\left|\frac{n r(r-1)}{2}-4 r+4-\left(\frac{n r(r-1)}{2}-4 r+6\right)\right| \frac{n r(r-2)}{2} \\
& +\left|0-\left(\frac{n r(r-1)}{2}-4 r+6\right)\right| \\
& +\sum_{i=2}^{n}\left|\frac{n r(r-1)}{2}-r+\lambda_{i}+2-\left(\frac{n r(r-1)}{2}-4 r+6\right)\right| \\
& +\left|\frac{n r(r-1)}{2}-2 r+2-\left(\frac{n r(r-1)}{2}-4 r+6\right)\right| \frac{n(r-2)}{2}
\end{aligned}
$$




$$
\begin{aligned}
& +\left|\frac{n r(r-1)}{2}-4 r+6-\left(\frac{n r(r-1)}{2}-4 r+6\right)\right| \frac{n r(r-2)}{2} \\
& +\left|2-\left(\frac{n r(r-1)}{2}-4 r+6\right)\right| \\
& =\sum_{i=2}^{n}\left|\lambda_{i}+3 r-6\right|+|2 r-6| \frac{n(r-2)}{2} \\
& +|-2| \frac{n r(r-2)}{2}+\left|\frac{-n r(r-1)}{2}+4 r-6\right|+\sum_{i=2}^{n}\left|\lambda_{i}+3 r-4\right| \\
& +|2 r-4| \frac{n(r-2)}{2}+|0| \frac{n r(r-2)}{2}+\left|\frac{-n r(r-1)}{2}+4 r-4\right| .
\end{aligned}
$$

All adjacency eigenvalues of a regular graph of degree $r$ satisfy the condition $-r \leq$ $\lambda_{i} \leq r, i=1,2, \ldots, n[5]$. Therefore if $r \geq 3$, then $\lambda_{i}+3 r-6 \geq 0, \lambda_{i}+3 r-4 \geq 0$, $2 r-6 \geq 0,2 r-4 \geq 0,(-n r(r-1) / 2)+4 r-6<0$, and $(-n r(r-1) / 2)+4 r-4<0$. Then from Eq. (4.12), and bearing in mind that $\sum_{i=2}^{n} \lambda_{i}=-r$, we get

$$
\begin{aligned}
L E\left(\overline{L^{2}(G)} \times K_{2}\right)= & \sum_{i=2}^{n} \lambda_{i}+(n-1)(3 r-6)+(r-3) n(r-2)+n r(r-2) \\
& +\frac{n r(r-1)}{2}-4 r+6+\sum_{i=2}^{n} \lambda_{i}+(n-1)(3 r-4) \\
& +(r-2) n(r-2)+\frac{n r(r-1)}{2}-4 r+4 \\
= & 2(n r-4)(2 r-3)-4 .
\end{aligned}
$$

Corollary 4.2. Let $G$ be a regular graph of order $n_{0}$ and of degree $r_{0} \geq 3$. Let $n_{k}$ and $r_{k}$ be the order and degree, respectively of the $k$-th iterated line graph $L^{k}(G)$ of $G$, $k \geq 2$. Then

$$
\begin{aligned}
L E\left(\overline{L^{k}(G)} \times K_{2}\right) & =\left(n_{k-2} r_{k-2}-4\right)\left(4 r_{k-2}-6\right)-4 \\
& =\left(2 n_{k-1}-4\right)\left(2 r_{k-1}-2\right)-4 \\
L E\left(\overline{L^{k}(G)} \times K_{2}\right) & =\left[\frac{n_{0}}{2^{k-2}} \prod_{i=0}^{k-2}\left(2^{i} r_{0}-2^{i+1}+2\right)-4\right]\left(2^{k} r_{0}-2^{k+1}+2\right)-4
\end{aligned}
$$




$$
L E\left(\overline{L^{k}(G)} \times K_{2}\right)=\frac{8 n_{k} r_{k}}{2+r_{k}}-4\left(r_{k}+1\right) .
$$

From Eq. (4.13) we see that the energy of $\overline{L^{k}(G)} \times K_{2}, k \geq 2$ is fully determined by the order $n$ and degree $r \geq 3$ of $G$.

Theorem 4.6. Let $G_{1}$ and $G_{2}$ be two Laplacian non-cospectral, regular graphs of the same order and of the same degree $r \geq 4$. Then for any $k \geq 2, L^{k}\left(G_{1}\right) \times K_{2}$ and $L^{k}\left(G_{2}\right) \times K_{2}$ is a pair of Laplacian non-cospectral, Laplacian equienergetic graphs possessing same number of vertices and same number of edges.

Proof. If $G$ is any graph with $n$ vertices and $m$ edges, then $G \times K_{2}$ has $2 n$ vertices and $2 m+n$ edges. Hence by repeated applications of Eqs. (4.1) and (4.2), $L^{k}\left(G_{1}\right) \times K_{2}$ and $L^{k}\left(G_{2}\right) \times K_{2}$ have same number of vertices and same number of edges. By Eqs. (4.5) and (4.6), if $G_{1}$ and $G_{2}$ are not Laplacian cospectral, then $L^{k}\left(G_{1}\right) \times K_{2}$ and $L^{k}\left(G_{2}\right) \times K_{2}$ are not Laplacian cospectral for all $k \geq 1$. Finally, Eq. (4.8) implies that $L^{k}\left(G_{1}\right) \times K_{2}$ and $L^{k}\left(G_{2}\right) \times K_{2}$ are Laplacian equienergetic.

Theorem 4.7. Let $G_{1}$ and $G_{2}$ be two Laplacian non-cospectral, regular graphs of the same order and of the same degree $r \geq 3$. Then for any $k \geq 2, \overline{L^{k}\left(G_{1}\right)} \times K_{2}$ and $\overline{L^{k}\left(G_{2}\right)} \times K_{2}$ is a pair of Laplacian non-cospectral, Laplacian equienergetic graphs possessing same number of vertices and same number of edges.

Proof. The proof is similar to that of Theorem 4.6 by using Eqs. (4.10), (4.11), and (4.13).

Acknowledgments. H. S. Ramane is thankful to the University Grants Commission (UGC), Govt. of India, for support through research grant under UPE FAR-II grant No. F 14-3/2012 (NS/PE). G. A. Gudodagi is thankful to the Karnatak University, Dharwad, for support through UGC-UPE scholarship No. KU/SCH/UGC-UPE/2014$15 / 901$.

\section{REFERENCES}

[1] E. O. D. Andriantiana, Laplacian energy, in: I. Gutman, X. Li (Eds.), Graph Energies - Theory and Applications, Univ. Kragujevac, Kragujevac, 2016, pp. 49-80.

[2] R. Balakrishnan, The energy of a graph, Linear Algebra Appl. 387 (2004), 287-295.

[3] F. Buckley, Iterated line graphs, Congr. Numer. 33 (1981), 390-394.

[4] F. Buckley, The size of iterated line graphs, Graph Theory Notes N. Y., 25 (1993), 33-36.

[5] D. Cvetković, M. Doob, H. Sachs, Spectra of Graphs - Theory and Application, Academic Press, New York, 1980.

[6] G. Dahl, The Laplacian energy of threshold graphs and majorization, Linear Algebra Appl. 469 (2015), 518-530.

[7] K. C. Das , S. A. Mojallal, Relation between energy and (signless) Laplacian energy of graphs, MATCH Commun. Math. Comput. Chem. 74 (2015), 359-366.

[8] K. C. Das, S. A. Mojallal, I. Gutman, On Laplacian energy in terms of graph invariants, Appl. Math. Comput. 268 (2015), 83-92.

[9] P. J. Davis, Circulant Matrices, Wiley, New York, 1979. 
[10] E. Fritscher, C. Hoppen, V. Trevisan, Unicyclic graphs with equal Laplacian energy, Lin. Multilinear Algebra 62 (2014), 180-194.

[11] H. A. Ganie, S. Pirzada, E. T. Baskoro, On energy, Laplacian energy and p-fold graphs, El. J. Graph Theory Appl. 3 (2015), 94-107.

[12] I. Gutman, E. Milovanović, I. Milovanović, Bounds for Laplacian-type graph energies, Miskolc Math. Notes 16 (2015), 195-203.

[13] I. Gutman, B. Zhou, Laplacian energy of a graph, Linear Algebra Appl. 414 (2006) 29-37.

[14] F. Harary, Graph Theory, Addison-Wesley, Reading, 1969.

[15] X. Li, Y. Shi, I. Gutman, Graph Energy, Springer, New York, 2012.

[16] B. Mohar, The Laplacian spectrum of graphs, in: Y. Alavi, G. Chartrand, O. R. Ollermann, A. J. Schwenk (Eds.), Graph Theory, Combinatorics and Applications, Wiley, (1991), pp. 871-898.

[17] S. Pirzada, H. A. Ganie, On the Laplacian eigenvalues of a graph and Laplacian energy, Linear Algebra Appl. 486 (2015), 454-468.

[18] S. Radenković, I. Gutman, Total $\pi$-electron energy and Laplacian energy: How far the analogy goes?, J. Serb. Chem. Soc. 72 (2007), 1343-1350.

[19] H. S. Ramane, S. B. Gudimani, Laplacian polynomial and Laplacian energy of some cluster graphs, Int. J. Sci. Innov. Math. Res. 2 (2014) 448-452.

[20] H. S. Ramane, I. Gutman, H. B. Walikar, S. B. Halkarni, Equienergetic complement graphs, Kragujevac J. Sci. 27 (2005), 67-74.

[21] H. S. Ramane, H. B. Walikar, S. B. Rao, B. D. Acharya, P. R. Hampiholi, S. R. Jog, I. Gutman, Spectra and energies of iterated line graphs of regular graphs, Appl. Math. Lett. 18 (2005), 679-682.

[22] H. Sachs, Über selbstkomplementare Graphen, Publ. Math. Debrecen 9 (1962), 270-288.

[23] H. Sachs, Über Teiler, Faktoren und charakteristische Polynome von Graphen, Teil II, Wiss. Z. TH Ilmenau 13 (1967), 405-412.

[24] D. Stevanović, Large sets of noncospectral graphs with equal Laplacian energy, MATCH Commun. Math. Comput. Chem. 61 (2009), 463-470.

\footnotetext{
${ }^{1}$ Department of Mathematics,

KARNATAK UNIVERSITY,

DHARWAD - 580003, INDIA

E-mail address: hsramane@yahoo.com

E-mail address: gouri.gudodagi@gmail.com
}

${ }^{2}$ FACUlty of SCIEnCE,

University of KraguJEVAC,

Kragujevac, Serbia, And

State University of Novi Pazar,

Novi Pazar, SERBia

E-mail address: gutman@kg.ac.rs 